\title{
The effect of teacher-made simulation moulage on learning cricothyrotomy skills in emergency medicine physicians
}

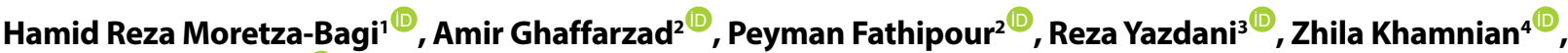 \\ Sama Rahnemayan ${ }^{5^{*}(\mathbb{D})}$
}

'Emergency Medicine Research Team, Tabriz University of Medical Science, Tabriz, Iran ${ }^{2}$ Department of Emergency Medicine, Tabriz University of Medical Sciences, Tabriz, Iran ${ }^{3}$ Trauma and Medical Emergencies Research Center, Hormozgan University of Medical Sciences, Bandar Abbas, Iran ${ }^{4}$ Department of Community Medicine, Tabriz University of Medical Sciences, Tabriz, Iran ${ }^{5}$ Student Research Committee, Tabriz University of Medical Sciences, Tabriz, Iran

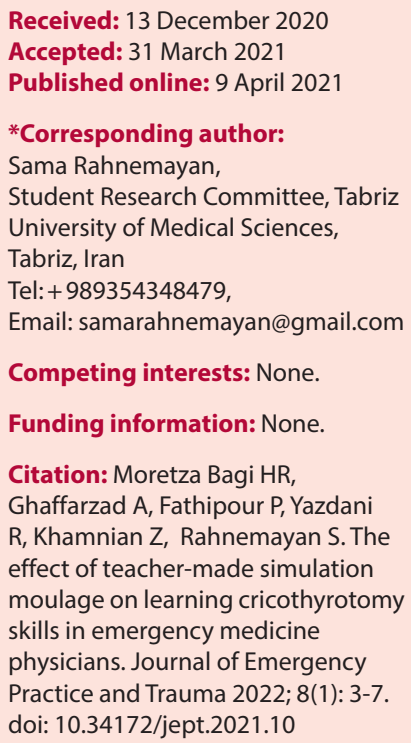

\begin{abstract}
Objective: Nowadays, simulation of clinical environment in medical education system (simulation-based learning) has led to a huge revolution in the quality of education and has increased the safety of educators and patients. In this study, we investigated the effect of teacher-made neck and lung simulators in teaching cricothyrotomy skills for emergency medicine residents.

Methods: In this pre-post test study, all faculty member of emergency medicine of Tabriz University of medical sciences specialty were invited to participate. After holding an educational and training session for assistants on a teacher-made moulage, all emergency medicine residents performed a tracheostomy on the commercial moulages of the skill lab unit for the second time and their scores were recorded.

Results: In this study, 23 emergency medicine residents participated. The mean \pm standard deviation of age was $35.91 \pm 3.57$ years. There was a significant difference between the mean duration of cricothyrotomy before and after the training $(P$ value $=0.006)$. There was also a significant difference between the mean scores obtained by residents in the preand post-training evaluation $(P$ value $<0.001)$

Conclusion: Findings showed that the moulages constructed by teachers not only can be effective in improving the cricothyrotomy skills in emergency medicine residents but also can reduce the likelihood of failure in performing cricothyrotomy.

Keywords: Cricothyrotomy, Difficult airway, Teacher made models, Skills laboratory, Emergency medicine
\end{abstract}

\section{Introduction}

Cricothyrotomy was first introduced in anesthesia literature by Fischer as an in-hospital alternate airway for the management of difficult airway (1). Tracheal intubation difficulties are the most common cause of brain damage or death from anesthesia, although they are not frequent. There are no exact clinical indications for the use of alternate airway. However, it is basically recommended in difficult or impossible situations for performing endotracheal intubation attempts or following failed endotracheal intubation attempts. "Difficult Airway" is defined as conditions involving severe airway trauma, difficult airway anatomy, or inadequate operator skill, which could be identified before performing initial intubations attempts, or after that. In situations where it is obviously difficult to perform airway management regardless of the skill of the operator, it is recommended to consider alternate airway management. When performing airway management, only three intubation attempts (insertion of blade) are recommended based on difficult airway studies and any extra endotracheal intubation attempts should be stopped (2,3).

It is very important to learn the skills of alternate airway management because of their use under urgent conditions, and simulated experiences are required just as well as practical experiences for training these skills (4-6). It seems quite necessary to learn this procedure on patients in controlled settings; however it may not be practical for most of alternate airway managements since they do not occur frequently in the emergency departments (6). 
Today, simulation of the clinical environment in the medical education system (simulation-based learning) has led to a huge revolution in the quality of education and has increased the safety of educators and patients. Medical directors believe that it is essential to use available and more efficient alternate airways in order to have an adequate training system (4,7-10). In this study, we investigated the effect of teacher-made simulators in teaching cricothyrotomy skills to emergency medicine residents.

\section{Methods}

This pre-post test study was conducted in the Skill Lab of Tabriz University of medical sciences in the year 2017 . study was approved by the ethics committee of Tabriz University of Medical Sciences with code number: IR.TBZMED. REC.1396. All emergency residents $(n=23)$ participated in this study. Emergency medicine residents who had previously learned the practice of cricothyrotomy were excluded as the expert resident. Based on the inclusion and exclusion criteria (the participant who has not interested to participate in the study or not complete the course was excluded), all emergency medicine residents initiated tracheostomy on the Commercial moulages in the skill lab unit at the faculty of medicine using the Direct Observation of Procedural Skills (DOPS) form, based on the American College of Surgeons (ACS) guidelines and their scores were recorded (11). The cricothyrotomy moulage was made of a gypsum mold along with poly vinyl chain (PVC) and silicon materials for the neck and 2 balloons resembling the lungs. Its simple structure comparing to other moulages was thought to be more cost effective and easier to work with. Figure 1 shows the consisting parts of the moulage. Cricothyrotomy was surgically taught by one of the emergency medicine professors on a teacher made moulage during a 2-hour training session for all assistants. In order to increase participation, the teacher's Moulage was provided to the residents for 1 hour and they were asked to do the necessary exercises to perform a cricothyrotomy without the presence of a teacher. After conducting the educational and training session for residents in coordination with the skill lab unit of the medical school, all emergency medicine residents performed a tracheostomy on the commercial moulages of the skill lab unit at the faculty of medicine under the form of DOPS and based on the ACS instruction and their scores were recorded. Data were analyzed using descriptive statistics (including tables and calculation of statistical indices of mean and standard deviation) and analytical statistics (linear regression, independent $t$ test) using SPSS software version 15.0.

\section{Results}

In this study of the 31 emergency medicine residents who enrolled in the study, 8 of them were excluded due to failure of completing all stages of the study or

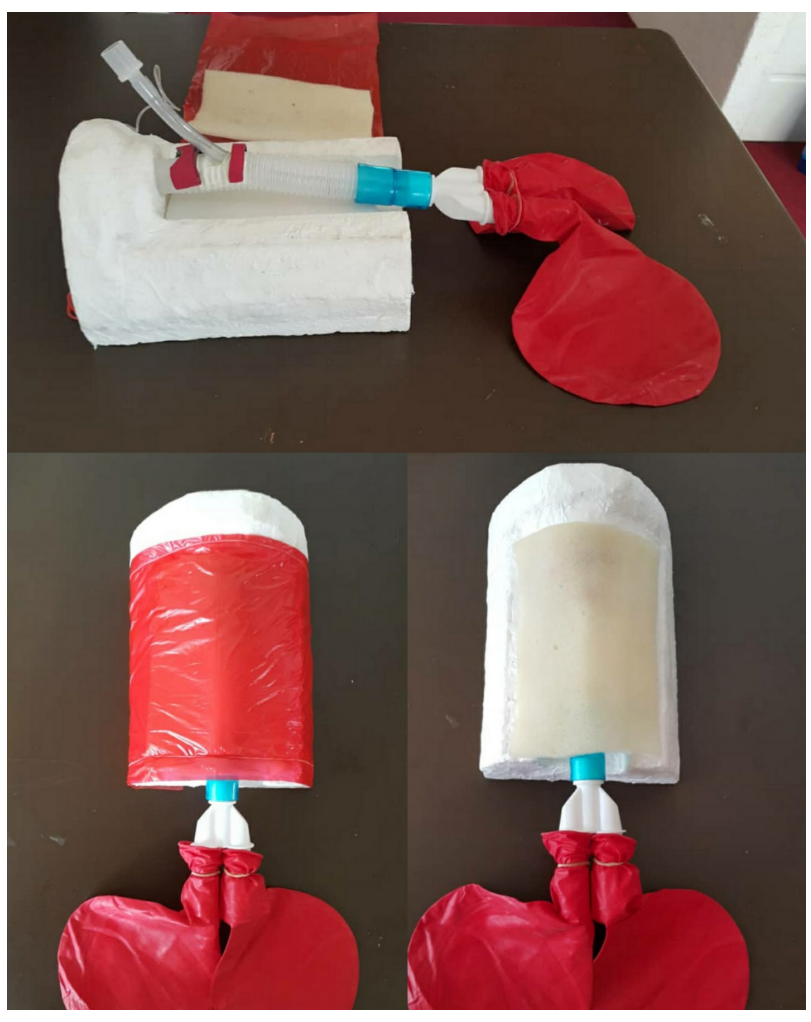

Figure 1. Teacher-made cricothyrotomy moulage.

refusing to participate in the study. Finally, 23 residents entered the study. Among this batch, 16 were males and 7 were females. The mean \pm standard deviation of age was $35.91 \pm 3.57$ years. The maximum of age was 42 years and the minimum age was 28 years.

All residents were evaluated using the cricothyrotomy checklist prior to training. The mean \pm standard deviation of the score obtained by the residents prior to the training session was $25.78 \pm 3.10$, with a maximum score of 32 and minimum of 20 .

After conducting the training session using a teacher made moulage, and providing the teacher made moulage for the training of the residents, a re-evaluation was done using a cricothyrotomy checklist. The mean \pm standard deviation of the score obtained after the training of the residents was $29.60 \pm 2.99$ with the maximum score of 35 and the minimum score of 25 .

Evaluation of cricothyrotomy duration before the educational session showed that the mean \pm standard deviation of the cricothyrotomy duration was $3.53 \pm 1.02$ min, with a maximum duration of 5 minutes and 30 seconds and a minimum time of 1 minute and 54 seconds.

After conducting the educational session and during cricothyrotomy, the duration of cricothyrotomy was re-recorded. The mean \pm standard deviation of the cricothyrotomy duration in the patients after the training session was $2.75 \pm 0.48$ minutes. The maximum duration was 3 minutes and 48 seconds and the minimum duration was 1 minute and 36 seconds. By comparing the duration of cricothyrotomy before and after cricothyrotomy 
training, findings showed that there was a significant difference between the mean duration of cricothyrotomy before and after training $(P$ value $=0.006)$. The results of cricothyrotomy duration and the checklist score of participants are presented in Table 1.

Based on the analysis of the scores obtained before and after cricothyrotomy training on the teacher made moulages in the two groups, findings showed a significant difference between the mean scores obtained by residents in the pre- and post-training evaluation ( $\mathrm{P}$ value $<0.001$ ). Thus, the difference between the mean score before and after the training was 3.82 , indicating an increase in the skill of residents on average, in at least 4 items out of the 18 items examined.

There was no significant relation between the pre- and post-training grade and the duration of cricothyrotomy before and after training and sex in all of the mentioned cases (all $P$ values were more than 0.05 ). This indicates that there is no difference in the improvement of task score or reduction in the duration of cricothyrotomy regarding the genders.

\section{Discussion}

The cricothyrotomy involves the formation of an upper airway skin cavity at the easiest and fastest available level, on the cricothyrotomy membrane, through which a respiratory cannula attaches to the ventilation system. Cricothyrotomy is used in the emergency department settings for primary and alternate airway management of difficult airways (12). Its use in the pre-hospital settings has been described in several studies $(3,13)$. The prehospital use of cricothyrotomy is not frequent due to its complications and poor outcomes; although it is being widely taught. $(14,15)$.

Schaumann et al assumed that training on cadavers and mannequins with different methods of cricothyrotomy is important for residents to act more effectively in reallife situations (16). A study by Metterlein et al indicated that wire-guided method shows fewer complications and therefore is a more reliable technique in comparison to other techniques for alternate airway managements, despite the fact that it is faster to perform direct puncture. However, both methods would provide sufficient ventilation when placed accurately (17). Aneeshkumar et al reported that cricothyrotomy is a simple, quick and safe procedure if there is adequate prior training for it, which provides efficient ventilation in emergency situations and

Table 1. Cricothyrotomy duration and the checklist ${ }^{\mathrm{a}}$ score of the participants

\begin{tabular}{lccc}
\hline & Before $($ mean \pm SD) & After $($ mean \pm SD) & $\boldsymbol{P}$ value \\
\hline $\begin{array}{l}\text { cricothyrotomy } \\
\text { checklist score }\end{array}$ & $25.78 \pm 3.10$ & $29.60 \pm 2.99$ & $<0.001$ \\
$\begin{array}{l}\text { cricothyrotomy } \\
\text { duration }\end{array}$ & $3.53 \pm 1.02$ & $2.75 \pm 0.48$ & 0.006 \\
\hline
\end{tabular}

acheck list score was according to step by step doing procedure which was written in ATLS guideline) results in no significant cardio-respiratory complications (18). In this study, we observed whether cricothyrotomy could be performed in emergency situations quickly and easily.

In a study by Shetty et al examining the ability to perform cricothyrotomy, it was shown that at least 5 attempts should be made to perform the cricothyrotomy on the pulp to acquire the skills required to perform the correct procedure (19).

Mandell and Orebaugh created a task simulator using the porcine larynx and an overlying animal skin to train anesthesiology residents for performing cricothyrotomy. The aim of the study was to create an effective, low-cost simplified trainer to simulate the airway and facilitate identification of the cricothyroid membrane with ultrasonography. They concluded that using this type of simulator provides a realistic partial-task trainer for open and percutaneous surgical airway education (20).

Aho et al developed another novel cricothyrotomy task trainer, constructed from household items. The model was constructed using a toilet paper roll, cardboard, Styrofoam, zip tie, and fabric to simulate the whole anatomic neck area; which was an ideal simulator for providing basic skills to all physicians in training, especially in settings with limited clinical opportunities and resources (21).

Hughes et al. developed another kind of cricothyrotomy task trainer using a three-dimensional printer and pioneering bleeding tissue to improve the trainer's realism and fidelity and concluded that their task trainer has some superiority comparing to the other non-bleeding models, providing an advanced platform to teach the critical and yet infrequent skill of cricothyrotomy (22).

One of the most important factors when considering different models is their cost, which make their repetition and program-wide use possible if not too expensive. Although various modalities have been described for cricothyrotomy, they are not always sustainable due to their cost (23-28). Using animal models as an alternative to real patients might be a good idea; however the access is limited and quite expensive. High-fidelity simulators as described above, have been developed to sidestep the limited access to animal models, however their cost remains as a barrier for their sustainability instead (24). The benefits of high vs. low-fidelity models are still unclear, however it is believed that low-fidelity cricothyrotomy simulations may be the same as high-fidelity simulations for gaining procedural skills $(24,28)$. It is not very common to have multiple attempts on a cadaver or animal model, but repetition is necessary for the formation of memory and skills.

Due to the fact that in our study the moulages were fully provided to residents, the number of attempts was not evaluated, so judgment about the amount of exercise and the skills required in this study could not be evaluated.

Also due to the setting and design of this study, it is possible that some increases in skills may be due to the additional training. Therefore, more studies with other 
possible settings are needed to gain more adequate results. On the other hand, in the present study, considering that all stages were performed on volunteers, the possible complications of cricothyrotomy therapy such as bleeding, hematoma and the failure of proper oxygenation were not evaluated.

\section{Conclusion}

The present study showed that the teacher constructed moulages can be effective in improving the cricothyrotomy skills in emergency medicine residents due to its simple structure. Also, since this moulage can be simply manufactured, it can be made by residents themselves for more additional training and reduce the time needed for participation in courses regarding busy curriculums and time constraint for the educators. In addition, using the teacher-made moulage for the basic training before using the moulage can result in better management of cricothyrotomy. Besides, the use of moulage can reduce the likelihood of failure in performing cricothyrotomy.

In summary, several studies using human simulators and cadavers have demonstrated the practicability of this technique for pre-hospital applications. There are limited descriptions of the method for clinical pre-hospital performance of cricothyrotomy; but this study shows that this method has the potential to be used as a simple technique to overcome difficult airway management in emergency departments.

\section{Authors' contributions}

HRMB conceived the idea or hypothesis for this research. AGh came up with the methodology to reach the conclusion. HRMB organized and supervised the course of the project. PF took responsibility in the execution of the experiments, follow-up of patients, data management and reporting. ZhKh took responsibility in logical interpretation and presentation of the results. SR took responsibility in doing the literature review. SR was responsible in the construction of the whole manuscript. RY reviewed the article before submission not only for spelling and grammar but also for its intellectual content. All authors read and approved the manuscript.

\section{Ethical issues}

This study was approved by the ethics committee of Tabriz University of Medical Sciences with code number: IR.TBZMED.REC.1396 (The ethical board approval for the study was obtained). All cases of the Helsinki Declaration and the code of ethics of the National Committee of Medical Ethics were fully observed in this study.

\section{Acknowledgements}

We express our thanks to Professor Samad Shams Vahdati for his comments on the manuscript. Additionally, we thank skill laboratory of Tabriz University of Medical Sciences and emergency medicine research team of Tabriz
University of Medical Sciences for their collaboration on conducting this study (research code: 57801).

\section{References}

1. Soleimanpour H, Shams Vahdati S, Mahmoodpoor A, Rahimi Panahi J, Afhami MR, Pouraghaei M, et al. Modified cricothyroidotomy in skill laboratory. J Cardiovasc Thorac Res 2012; 4(3): 73-6. doi: 10.5681/jcvtr.2012.018.

2. Wang HE, Yealy DM. How many attempts are required to accomplish out-of-hospital endotracheal intubation? Acad Emerg Med 2006; 13(4): 372-7. doi: 10.1197/j. aem.2005.11.001.

3. Wang HE, Kupas DF, Greenwood MJ, Pinchalk ME, Mullins T, Gluckman W, et al. An algorithmic approach to prehospital airway management. Prehosp Emerg Care 2005; 9(2): 145-55. doi: 10.1080/10903120590924618.

4. Kneebone R. Simulation in surgical training: educational issues and practical implications. Med Educ 2003; 37(3): 267-77. doi: 10.1046/j.1365-2923.2003.01440.x.

5. Dent JA. Current trends and future implications in the developing role of clinical skills centres. Med Teach 2001; 23(5): 483-9. doi: 10.1080/01421590120075724.

6. Kozu T. Medical education in Japan. Acad Med 2006; 81(12): 1069-75. doi: 10.1097/01.ACM.0000246682.45610. dd.

7. Hao J, Estrada J, Tropez-Sims S. The clinical skills laboratory: a cost-effective venue for teaching clinical skills to third-year medical students. Acad Med 2002; 77(2): 152. doi: 10.1097/00001888-200202000-00012.

8. Kneebone R, Nestel D. Learning clinical skills-the place of simulation and feedback. Clin Teach 2005; 2(2): 86-90. doi: 10.1111/j.1743-498X.2005.00042.x.

9. Weller J, Robinson B, Larsen P, Caldwell C. Simulationbased training to improve acute care skills in medical undergraduates. N Z Med J 2004; 117(1204): U1119.

10. Paparella SF, Mariani BA, Layton K, Carpenter AM. Patient safety simulation: learning about safety never seemed more fun. J Nurses Staff Dev 2004; 20(6): 247-52. doi: 10.1097/00124645-200411000-00001.

11. Galvagno SM Jr, Nahmias JT, Young DA. Advanced trauma life support ${ }^{\oplus}$ update 2019: management and applications for adults and special populations. Anesthesiol Clin 2019; 37(1): 13-32. doi: 10.1016/j.anclin.2018.09.009.

12. Burkey B, Esclamado R, Morganroth $M$. The role of cricothyroidotomy in airway management. Clin Chest Med 1991; 12(3): 561-71.

13. Fortune JB, Judkins DG, Scanzaroli D, McLeod KB, Johnson SB. Efficacy of prehospital surgical cricothyrotomy in trauma patients. J Trauma 1997; 42(5): 832-6. doi: 10.1097/00005373-199705000-00013.

14. Wang J, Lin YI, Hou SY. A data mining approach for training evaluation in simulation-based training. Comput Ind Eng 2015; 80: 171-80. doi: 10.1016/j.cie.2014.12.008.

15. Buonopane CE, Pasta V, Sottile D, Del Vecchio L, Maturo A, Merola R, et al. Cricothyrotomy performed with the Melker $^{\text {res }}$ set or the QuickTrach ${ }^{\mathrm{Tw}}$ kit: procedure times, learning curves and operators' preference. G Chir 2014; 35(7-8): 165-70.

16. Schaumann N, Lorenz V, Schellongowski P, Staudinger T, Locker GJ, Burgmann H, et al. Evaluation of Seldinger 
technique emergency cricothyroidotomy versus standard surgical cricothyroidotomy in 200 cadavers. Anesthesiology 2005; 102(1): 7-11. doi: 10.1097/00000542-20050100000005.

17. Metterlein T, Frommer M, Ginzkey C, Becher J, Schuster F, Roewer N, et al. A randomized trial comparing two cuffed emergency cricothyrotomy devices using a wire-guided and a catheter-over-needle technique. J Emerg Med 2011; 41(3): 326-32. doi: 10.1016/j.jemermed.2010.04.008.

18. Aneeshkumar MK, Jones TM, Birchall MA. A new indicator-guided percutaneous emergency cricothyrotomy device: in vivo study in man. Eur Arch Otorhinolaryngol 2009; 266(1): 105-9. doi: 10.1007/s00405-008-0698-5.

19. Shetty K, Nayyar V, Stachowski E, Byth K. Training for cricothyroidotomy. Anaesth Intensive Care 2013; 41(5): 623-30. doi: 10.1177/0310057x1304100508.

20. Mandell D, Orebaugh SL. A porcine model for learning ultrasound anatomy of the larynx and ultrasound-guided cricothyrotomy. Simul Healthc 2019; 14(5): 343-7. doi: 10.1097/sih.0000000000000364.

21. Aho JM, Thiels CA, AlJamal YN, Ruparel RK, Rowse PG, Heller SF, et al. Every surgical resident should know how to perform a cricothyrotomy: an inexpensive cricothyrotomy task trainer for teaching and assessing surgical trainees. J Surg Educ 2015; 72(4): 658-61. doi: 10.1016/j. jsurg.2014.12.012.

22. Hughes KE, Biffar D, Ahanonu EO, Cahir TM, Hamilton A, Sakles JC. Evaluation of an innovative bleeding cricothyrotomy model. Cureus 2018; 10(9): e3327. doi: 10.7759/cureus.3327.

23. McCarthy MC, Ranzinger MR, Nolan DJ, Lambert CS, Castillo MH. Accuracy of cricothyroidotomy performed in canine and human cadaver models during surgical skills training. J Am Coll Surg 2002; 195(5): 627-9. doi: 10.1016/ s1072-7515(02)01337-6.

24. Friedman Z, You-Ten KE, Bould MD, Naik V. Teaching lifesaving procedures: the impact of model fidelity on acquisition and transfer of cricothyrotomy skills to performance on cadavers. Anesth Analg 2008; 107(5): 1663-9. doi: 10.1213/ane.0b013e3181841efe.

25. Eaton BD, Messent DO, Haywood IR. Animal cadaveric models for advanced trauma life support training. Ann R Coll Surg Engl 1990; 72(2): 135-9.

26. Sergeev I, Lipsky AM, Ganor O, Lending G, Abebe-Campino $\mathrm{G}$, Morose A, et al. Training modalities and self-confidence building in performance of life-saving procedures. Mil Med 2012; 177(8): 901-6. doi: 10.7205/milmed-d-12-00018.

27. Custalow CB, Kline JA, Marx JA, Baylor MR. Emergency department resuscitative procedures: animal laboratory training improves procedural competency and speed. Acad Emerg Med 2002; 9(6): 575-86. doi: 10.1111/j.15532712.2002.tb02294.x.

28. Cho J, Kang GH, Kim EC, Oh YM, Choi HJ, Im TH, et al. Comparison of manikin versus porcine models in cricothyrotomy procedure training. Emerg Med J 2008; 25(11): 732-4. doi: 10.1136/emj.2008.059014. 